\title{
L'essence du sport. Pour une approche anthropologique et métaphysique
}

\section{The essence of sport: An anthropological and metaphysical approach}

\author{
Fabrice LOUIS \\ $\mathrm{XXX}$ \\ CeDS, EA 7440 \\ AHP, UMR 7117
}

Résumé : Il s'agit de donner une analyse conceptuelle et une définition essentialiste de l'action sportive. D'autre part nous soutiendrons que les propriétés qu'on attribue à des actions sportives sont bien réelles. Nous opposons ces deux conceptions :

- CSH : conception socio-historique. Dans une telle conception, le sport est conçu comme l'ensemble des pratiques physiques reconnues ainsi, naissant, évoluant, disparaissant, trouvant une fonction et un sens avec un contexte social précis.

- CRE : conception réaliste et essentialiste du sport. Dans cette perspective, pratiquer du sport, consiste à s'engager physiquement, émotionnellement, de manière intense, dans le cadre d'une compétition qui résulte d'une action conjointe paradoxale et produit une œuvre unique.

Le sport reflète un trait de la nature humaine, celui d'une capacité à coopérer d'une manière nouvelle, à la manière dont nous avons appris à coopérer pour élaborer des actes de langage nouveaux et spécifiquement humains.

Il faut apprendre, non pas en quoi le sport peut nous être utile, ou bien en quoi il a de la valeur, mais tout simplement ce qu'est le sport.

MotS-CLÉs : sport, réalisme, essentialisme, philosophie, action conjointe

Aвstracr: The aim of this article is to provide a conceptual analysis and an essentialist definition of sport. I will also argue that the properties attributed to sporting activities are indeed real. I compare the following two differing conceptions of sport:

- SHC: socio-historical conception. In this conception, sport is conceived as the set of physical practices that emerge, evolve, disappear, and find a function and meaning within a specific social context.

- REC: realistic and essentialist conception of sport. From this perspective, practicing sport consists in intensely engaging, physically and emotionally, in a competition that results from a paradoxical joint action and produces a unique outcome.

Sport reflects a characteristic of human nature, that of an ability to cooperate in a new way, the way in which we have learned to cooperate to develop new and specifically human acts of language. Instead of learning how sport can be useful to us, or its value, we must simply learn what sport is.

Kerworos: sport, realism, essentialism, philosophy, joint action 


\section{INTRODUCTION}

De l'aveu même de certains sociologues (Vieille-Marchiset \& Tatu-Colasseau, 2015, p. 39), le sport étant à la croisée de nombreux champs, sa définition est « flottante, composite et métissée » et « il est justifié de se demander si une sociologie du sport est possible ». Dans cet article, nous donnons une définition essentialiste qui restreint considérablement la classe des activités relevant du sport. Nous assimilons alors les activités rejetées à celles qui figurent dans une autre classe, celle des " groupes frères » du sport, selon la classification biologique importée par Lesage (2015, p. 3454). Ces " groupes frères » recensent des pratiques particulièrement apparentées avec le sport (parce qu'elles nécessitent un intense engagement physique, parce qu'elles sont des pratiques ludo-motrices...) sans toutefois présenter l'ensemble des caractéristiques de l'activité sportive. Notre but est d'éliminer les définitions fondées sur les innombrables représentations sociales qui affaiblissent l'acuité avec laquelle nous devons envisager le sport.

Selon Parlebas (2015, p. 2218), « l'exemple le plus frappant d'une cacophonie sémantique est fourni par le terme "sport" ». À propos de la sociologie, Parlebas (2015, p. 2218) ajoute : «Il est stupéfiant qu'une discipline qui se veut scientifique n'ait pas réussi à définir de manière indiscutable son "objet" quand celui-ci correspond à un fait social d'une telle notoriété [...]. Cette carence autorise, de manière basique, à mettre dans la même catégorie la baignade d'un brave vacancier qui s'immerge au bord de la plage et la performance d'un finaliste de natation aux Jeux olympiques. »

Nous tenterons de dire ce qu'est le sport comme on peut se demander ce qu'est une œuvre d'art. Dans les deux cas, il est probable, comme le suggère Pouivet (1999, p. 7), qu'un « certain discrédit jeté sur l'idée même de prétendre dire ce que sont les choses en elles-mêmes ait conduit les philosophes à se détourner de l'interrogation ontologique et métaphysique en général. Ce discrédit résulte, semble-t-il, de l'importance prise par des courants philosophiques qui mettent l'accent sur le sujet connaissant ou moral plutôt que sur l'objet connu ou des vérités morales indépendantes de lui. On a beaucoup insisté sur l'emprise des modèles sociaux sur ce sujet trop sûr de son indépendance. »

Dans ce qui suit, la thèse anthropologique et métaphysique adoptée est celle de Pouivet (2017, p. 15) : " La métaphysique réaliste et finaliste proposée est générale : elle compose une compréhension de ce qu'est la réalité et de sa raison d'être. " La thèse est aussi anthropologique car elle «précise le statut métaphysique de l'être humain, comme producteur d'artefact et d'œuvre d'art » (Pouivet, 2017, p. 133). À la différence de la perspective sociologique qui explique des faits sociaux par des causes sociales et donc par d'autres faits sociaux, notre thèse lie de manière ontologique deux descriptions : celle qui se soucie de dire ce qu'est une vie proprement humaine et celle qui tente de dire ce qu'est une action réellement sportive. L'une ne va pas sans l'autre.

Nous soutiendrons que les propriétés sportives qu'on attribue à des actions lors d'une compétition sont bien réelles. Ce qui signifie qu'en décrivant l'action d'un compétiteur comme étant celle d'un sportif, nous affirmons quelque chose au sujet du monde, nous ne faisons pas seulement un énoncé reflétant nos représentations. Il s'agit ici d'une défense d'un réalisme en sport comme il en existe un pour l'esthétique ${ }^{1}$. Nous réfutons donc la conception relativiste du sport, au même titre que s'il s'agissait de définir un acte de langage comme l'acte de promettre ${ }^{2}$.

À propos des jeux sportifs nés en Grèce et de leur évolution sous l'influence romaine,

1 Tel que le défend par exemple Pouivet dans son Réalisme esthétique (2006).

2 On peut fort bien penser ce qu'on veut de l'acte de donner, tout en admettant que l'acte de donner ne dépend pas des conditions sociales dans lesquelles on donne. 
Gillet (1949, p. 38) déclare : « Les Romains, conquérants de la Grèce, ne comprirent pas que ces jeux, qu'ils empruntèrent à leurs vaincus, ne tenaient leur valeur que de la manière dont ils étaient pratiqués. » Selon Gillet, les jeux de balle n'étaient plus que des amusements, l'entraînement corporel n'était estimé que par l'avantage qu'il donnait aux légions et l'arène des gladiateurs n'était qu'un lieu de spectacle destiné à montrer toute la cruauté des hommes. Mais ce n'est pas seulement la valeur du sport que la civilisation des Romains perdait ainsi, c'est le concept du sport. Et si le désarroi des amoureux du sport moderne est aussi important, c'est que nous sommes en train de vivre ce que les Romains ont vécu. Nous pensons perdre parfois les valeurs du sport alors que nous sommes en train d'oublier le sens du concept, sous l'injonction sociale de rendre utile tout ce que nous faisons. Il nous faut réapprendre non pas en quoi le sport peut nous être utile mais tout simplement ce qu'est le sport. Apprendre pour connaître ce qu'est le sport et non pas seulement apprendre par le sport. Ce n'est pas qu'une question d'utilité sociale : le pratiquant sportif ne serait alors qu'un bon citoyen en véhiculant les valeurs prônées par ses contemporains. C'est une question éthique qui lie la pratique du sport avec l'exercice de vertus et avec la possibilité de se réaliser en tant qu'être humain. Ce sera l'objet de notre dernier paragraphe.

Selon Jeu (1993, p. 36), la définition du sport doit tenir compte " des valeurs antithétiques, victoire ou défaite, et cette dichotomie pèse sur le sport un peu comme le bien et le mal en morale, ou le vrai et le faux en logique ». Nous souscrivons à cette idée et pour cela, nous opposerons ces deux conceptions :

CRE : conception réaliste et essentialiste du sport. Dans cette perspective, pratiquer du sport consiste à s'engager physiquement, émotionnellement, de manière intense, dans le cadre d'une compétition éphémère qui résulte d'une action conjointe paradoxale et produit une œuvre unique.
CSH : conception socio-historique. Dans une telle conception, « le sport apparaît comme un phénomène historiquement déterminé, donc transitoire puisqu'il n'y a pas de traits permanents de la nature humaine impliqués dans sa définition " (Moreau \& Taranto, 2008, p. 28). Par conséquent, le sport est conçu comme l'ensemble des pratiques physiques reconnues ainsi, naissant, évoluant, disparaissant, trouvant une fonction et un sens avec un contexte social précis. Ainsi, « l'approche réduit le sport aux représentations qu'un échantillon de personnes s'en font » (Guay, 1993, p. 21).

Ce que nous allons tenter de montrer, c'est que le sport reflète un trait de la nature humaine, celui d'une capacité à coopérer d'une manière nouvelle, à la manière dont nous avons appris à coopérer pour élaborer des actes de langage nouveaux et spécifiquement humains. Par conséquent, la question se pose de savoir s'il serait possible qu'un nombre important de personnes puisse croire qu'une activité soit sportive, et qu'en réalité elle ne le soit pas. La thèse que nous tentons de réfuter répond non, par principe.

\section{De L'UTILITÉ dóUNE déFinition ÉCHAPPANT A L'APPROCHE SOCIO-HISTORIQUE : LE SPORT SANS SES ORIPEAUX}

Rauch (1970, p. 24) admet, pour critiquer la prégnance du sport dans nos sociétés modernes, un postulat que nous allons défendre par la suite : " Le système éthique de l'action sportive [...] devient la nature même de cette activité, faute de quoi celle-ci perd, non seulement sa fonction normative, mais son contenu même. » Il est vrai que, sans éthique, "l'activité athlétique se dépouille de sens " (Rauch, 1970, p. 24). Mais cette contrainte n'est pas liée à la fonctionnalité du sport, fonctionnalité qui serait propre à nos sociétés. Il s'agit, comme nous le montrerons, d'une question d'anthropologie et de logique. Il y a bien 
quelque chose d'absolu dans l'éthique de l'action sportive, comme le remarque Rauch (1970, p. 23).

Il est nécessaire d'échapper à une définition trop générale où le sport « englobe tout type d'activité physique réalisée dans un but récréatif, hygiénique ou compétitif, dans un cadre réglementaire minimal » (Terret, 2010, p. 10) afin de saisir que "l'esprit " du sport n'est pas un attribut facultatif de l'action des sportifs. Il ne distingue pas les sportifs vertueux des autres, il constitue l'essence même de toute action sportive.

En faisant abstraction des structures sociales dans lesquelles les activités sportives naissent et se développent, en faisant abstraction de ce que l'homme projette sur son activité, nous pourrons définir le sport comme une activité qui ne se confondra plus avec d'autres formes d'activités ayant comme " un air de famille » (Wittgenstein, 2004, §67) avec le sport. Ces activités cousines sont d'ailleurs le plus souvent affublées d'adjectifs : sport-spectacle, sport professionnel, sport loisir, sports bien-être, e-sports. Beaucoup d'activités ont été confondues avec le sport parce qu'elles ont des caractères communs avec le sport. Elles présentent par exemple un caractère ludique ou bien un engagement physique important. Les raisons pour lesquelles les représentations du sport évoluent tiennent à sa composante anthropologique : il est vrai que le sport émerge à partir d'autres activités humaines comme la chasse, la guerre, le jeu, la locomotion, l'utilisation d'outils... Or nous ne verrons apparaître la nature du sport qu'en nous débarrassant de ce qui le travestit : les multiples intérêts que les uns et les autres portent à toutes les activités qui gravitent autour. Avec une définition faisant table rase de tout ce qui ressemble au sport, nous n'éliminerons pas le dopage, la discrimination, l'utilisation mercantile ou politique, le sexisme... Mais il deviendra évident que ces pratiques condamnables touchent les activités humaines qui n'ont d'apparence sportive que pour ceux qui ignorent l'essence du sport ou ceux qui tentent d'abuser les jeunes pratiquants. Car faire croire que l'action sportive contient en son sein, par nature, l'ombre du dopage est désastreux sur un plan éducatif. Il est au contraire nécessaire de faire comprendre que, par nature, un sportif ne peut se doper. «Sportif dopé » est un oxymore.

Il est probable que cet oxymore n'apparait pas tant qu'on ne fait pas la différence entre différents types d'apprentissages : ceux qui peuvent être réalisés de manière non intentionnelle (apprendre à se relever après une chute, apprendre à attraper un objet) et ceux qui ne peuvent être faits qu'en ayant accès à l'exacte description de ce qu'il convient d'être ou de faire. On ne peut se marier sans savoir ce qu'on doit faire. On ne peut pas être catholique si on ne saisit pas le sens des actions de l'homme d'Église donnant l'hostie. De même, on ne peut être sportif qu'en ne se trompant pas sur le sens qu'il convient d'attribuer à ses propres actions. Par conséquent, se doper ou être sportif : il faut choisir.

\section{L'APPROCHE SOCIOLOGIQUE ET LA CRITIQUE MARXISTE DU SPORT}

Les fonctions sociales (intégrer un groupe de personnes par le sport, améliorer sa santé, honorer sa patrie...) ne définissent pas le type d'action qu'est l'action sportive, pas plus que l'utilité sociale de l'acte de promettre ne définit pas l'acte de promettre. Prenons l'exemple du mariage : il est utile de connaitre la place que tient le mariage dans une société en étudiant les représentations qu'ont les membres, la place que le mariage tient par rapport à l'union libre, au PACS. Mais cela ne définit pas encore l'acte de se marier. On peut de même, selon Vigarello (2003, p. 185), «approfondir le spectre des sports en le fondant sur des goûts sociaux, des modes d'être, des sensibilités collectives, tous susceptibles de guider les choix ». Connaître la prédominance de tel ou tel sport chez les ouvriers ou les cadres supérieurs est 
sans doute utile pour lancer une campagne de publicité bien ciblée mais cela ne nous permet pas de comprendre ce que le sportif éprouve en pratiquant une activité sportive.

Lactivité sportive a un air de famille avec de nombreuses activités qui ont été utiles à l'humanité pour survivre. Cet aspect anthropologique est fondamental et peut facilement être mal interprété pour une raison qui apparaîtra avec la critique marxiste du sport. Il y a plus de quarante ans, Brohm (1976, pp. 320321) écrivait : "Les performances sportives sont de pures dépenses évanescentes, éphémères, qui ne se trouvent jamais à se concrétiser dans un produit, dans une œuvre durable [...]. L'institution sportive est une pétrification idéologique spécifique qui participe au maintien de l'ordre bourgeois. » Cette critique était fondée sur une étude des fonctions des activités humaines. Quelles fonctions a le sport dans nos sociétés capitalistes ? Quel rôle occupe-t-il parmi toutes les autres institutions? La réponse de Brohm (1976, pp. 318-319) était sans appel : «Le sport est le code du corps dans un système cybernétique [...]. Le sport, avons-nous dit, est le behaviorisme symbolique d'une société capitaliste industrielle. » Quarante ans après, la critique a évolué mais elle reprend le même type d'arguments, comme le montre l'interprétation d'Ollier (2010) : « C'est toujours le même scénario, les matches sont prévisibles, les violences, les problèmes de dopage et de surentraînement, et enfin la récupération politique, idéologique, commerciale. Tout est lié et il est impossible de dissocier ces éléments. »

Cette perspective sociologique peut être soumise à la même critique que celle d'un naturalisme qui réduirait un être vivant au fonctionnement de ses organes. Un être vivant est une forme dynamique qui unifie l'ensemble de ses organes. Réduire l'être vivant au fonctionnement de son corps, c'est oublier le caractère essentiellement finalisé de l'activité humaine. De la même manière, l'activité sportive, qui est caractéristique des êtres humains à une certaine période de l'humanité, dispose d'un corps social dont elle se sert d'une manière plus ou moins heureuse pour exister. La publicité dans le sport, le goût du jeu, l'ambition, la gloire, les conquêtes amoureuses après la victoire, le culte du corps, l'argent, la domination, le sentiment d'invulnérabilité... sont autant d'« organes " qui fonctionnent pour qu'existe le sport. Mais réduire le sport au fonctionnement d'organes issus d'un corps social, c'est négliger complètement ce qui est le fondement de toute existence, l'aspiration à une forme d'harmonie.

Que cette aspiration soit affaiblie par une hypertrophie de certains des " organes sociaux " qui permettent au sport d'exister ne doit pas nous amener à nous tromper sur la véritable nature du sport. L'activité sportive est une sorte d'exaptation d'autres activités humaines. En modifiant le sens qu'on accordait à certaines actions humaines (courir pour fuir ou attraper, lancer, nager, jouer) nous leur avons fait jouer un autre rôle dans nos sociétés. Bien entendu, comme dans le cas des exaptations naturelles, l'environnement joue un rôle sélectif. Mais cela n'implique pas que l'activité sportive soit simplement « la pétrification idéologique » d'un ordre bourgeois. Ce que le sportif ressent ne peut pas être compris par une analyse sociale du sport.

\section{Ce qui est Vivant et mort dans L'APPROCHE ARCHÉOLOGIQUE DU SPORT}

Les partisans d'une archéologie du sport tentent " de dégager des continuités et des ruptures, des altérations du mode d'être des choses offertes au savoir " (Gleyse, 2015, p. 330). L'idée qu'il y a une altération du mode d'être du sport est utile pour concevoir l'activité sportive comme ayant un mode d'existence qui n'est pas celui d'une chose inerte. L'activité sportive émerge au sein d'autres activités humaines, elle naît pourrait-on dire, engendrée par l'existence d'une nouvelle perspective de l'homme sur ses propres actions. Mais 
l'archéologue du sport considère que cette perspective n'est qu'un reflet qu'il convient d'analyser pour faire apparaitre la vraie réalité sociale. Ainsi, selon Foucault (1969, p. 218), "l'archéologie analyse le degré et la forme de perméabilité d'un discours ». Selon Gleyse (2015, p. 329), le sport et ses pratiques, dans cette perspective, véhiculent une épistémè, c'est-à-dire un ensemble de relations qui se déposent à travers eux et que l'on peut découvrir en étudiant les régularités discursives et la porosité du sport avec trois champs : « techniques laborieuses, sciences et pratiques corporelles et activités physiques». De sorte que « le sport, et plus largement les pratiques corporelles $₫$ ainsi que les discours sur le corps et les pratiques corporelles $₫$ permettraient de dévoiler, dans une perspective archéologique : la peau du réel ou du moins une certaine couche, l'épithélium de la peau du réel » (Gleyse, 2015, p. 328).

Cette forme d'étude repose en partie sur l'idée que « toute motricité est une ethnomotricité » (Parlebas, 1985, p. 26) et qu'elle évolue au rythme de la société dans laquelle elle a une fonction. Une rupture sociale engendre une rupture de l'usage des motricités. Le remplacement des paysans par des machines agricoles provoque la disparition de la motricité liée à l'emploi de la faux. Mais il est vrai aussi que des activités semblables sur un plan de la motricité peuvent prendre alors des significations différentes. Par exemple, il est possible de marcher sur le chemin de Saint-Jacques de Compostelle pour visiter la région ou au contraire pour faire un pèlerinage. Pour Parlebas (1985, p. 32) le jeu appelé tlachtli des Aztèques ne peut pas être pris pour l'ancêtre du basket car, même s'il y a une ressemblance forte des actions (marquer un panier avec un ballon), la symbolique est différente. Tout d'abord, défaite et victoire ont la même importance. Ensuite, chez les Aztèques, il s'agit de canaliser l'énergie pour l'utiliser socialement, comme un rite. On pourrait cependant répondre à Parlebas qu'il est possible de concevoir ainsi tous les sports, comme une manière de canaliser l'énergie et l'attention des sportifs et des spectateurs afin d'éviter des secousses sociales qui remettraient en cause l'ordre établi. Le sport, comme activité, ne serait donc qu'un marqueur de l'évolution de nos sociétés, comme les espèces vivantes le sont pour l'évolution des écosystèmes. Et de ce fait l'étude du sport ne serait intelligible que par une étude archéologique des sports.

Il y a ici une confusion entre forme et structure. Si je regarde les modifications anatomiques d'un arbre pendant une saison entière, je peux savoir dans quelle saison nous sommes, et connaitre l'influence des saisons sur l'arbre. Mais je ne dois pas oublier que l'arbre est d'abord une forme vivante qui a une dynamique interne. Considérer qu'il n'y a pas de «dynamique interne » (Gleyse, 2015, p. 329) au sport, c'est réduire considérablement l'étude anthropologique qu'on peut en faire. L'anthropologie n'est réellement intelligible qu'au travers d'une métaphysique de l'être humain. Il est cependant nécessaire de qualifier le type d'anthropologie dont il va être question. Cette étude est fondée sur l'existence d'ontogénies et non d'ontologies. Quel est l'objet de l'une et de l'autre ? La différence entre les deux est précisée par Ingold (2014, p. 74) : «Il ne s'agit pas de structures mais d'êtres en cours de générations ${ }^{3}$. » Lorsque nous tentons de comprendre ce qu'est le sport, nous devons tenter de comprendre les actions des êtres qui se développent par le sport. Il ne s'agit pas de s'arrêter aux structures sociales qui freinent ou accentuent ce développement. Que le tlachtli soit vécu de la même manière par le vaincu ou le vainqueur est permis par le fait que c'est un

3 La thèse défendue ici est plus précisément celle de Ruver et d'Aristote : les êtres humains actualisent des formes de manière finalisée. L'apparence que prend cette réalisation peut dépendre du type de société dans laquelle les êtres humains vivent. Mais cette contingence ne change en rien un certain mode d'existence : viser des fins et agir selon des thèmes propres à l'espèce, agir de manière vertueuse ou non, de manière harmonieuse, esthétique, sportive... 
rituel qui canalise les énergies des uns et des autres. Mais ce qui importe, c'est qu'il soit possible, à ce moment de l'évolution humaine, de vivre de la même manière le jeu, qu'on soit perdant ou vainqueur. L'étude anthropologique du sport se prête à l'analyse que fait Ingold des faits sociaux. L'anthropologue souscrit à l'analogie de Radcliff Brown : on peut comprendre le fait social comme un processus vital. Cette idée permet d'entrevoir de la continuité dans le changement. "Dans la vie, la forme n'est pas spécifiée de l'intérieur; elle ne cesse d'émerger et aucune chose n'est tout à fait la même d'un instant à l'autre " (Ingold, 2013, p. 316). Ainsi, la forme du sport est multiple et certaines formes, comme les espèces, disparaissent ou au contraire se développent. Mais ces évolutions sont le fruit de la vie, c'està-dire d'un principe qui est à l'origine des évolutions de formes sélectionnées par un milieu plus ou moins agressif. Qu'il soit utile de concevoir une sorte de darwinisme social pour concevoir certaines ruptures dans l'histoire du sport ne devrait pas faire masquer un fait pourtant évident : toute action est l'œuvre d'un ou plusieurs êtres humains qui évoluent eux aussi en même temps que les sociétés dans lesquelles ils vivent. Ne retenir dans l'œuvre sportive que la face sociale revient à s'interdire de décrire ce qui est apparu de nouveau dans l'existence humaine, quand le sport est apparu.

L'étude d'une activité sportive, de ses règles, de son évolution est différente de l'étude de ce qu'est l'activité sportive. Une activité sportive peut évoluer dans le temps mais le concept doit rester invariant dans le temps pour que l'idée d'évolution prenne un sens. La même erreur peut être faite à propos d'une étude de ce qu'est le jeu, étude qui serait confondue avec une étude des différents jeux. Ainsi raisonne Chauvier (2007, p. 18) pour faire la distinction entre « jouer à l'élastique » et " jouer au jeu de l'élastique » : ce qui est constitutif du jeu et pas de l'activité de jouer avec un élastique, c'est la règle du jeu. Décrire ce que sont les comportements ludiques n'est pas équivalent à la description de la logique d'un jeu car ce jeu est institutionnalisé par une règle qui contraint logiquement les comportements. Décrire le dispositif du jeu, c'est donner des critères permettant d'identifier les comportements horsjeu des autres comportements. De même, il est utile de faire la différence entre la description des comportements sportifs et la description de ce qu'est une action en tant qu'elle est sportive.

\section{UNE DÉFINITION ESSENTIALISTE DU SPORT}

Se poser la question de la nature de l'action sportive, c'est se poser la question des critères qui permettent de distinguer le sportif de ceux qui n'en prennent que l'apparence. Celui qui chausse ses baskets pour aller courir imite simplement la phase préparatoire de l'athlète avant la compétition. Il ne peut être pris pour un sportif que si on admet que la finalité du sport est "d'orienter et de canaliser une pure dépense d'énergie physique " (Haumesser, 2008, p. 69).

Laction sportive a deux dimensions. La première dimension caractérise un certain type d'actions : les actions conjointes qui sont par nature relationnelles et témoignent d'une relation bien particulière. L'action sportive est, selon cette dimension, caractérisée par un critère externe à l'agent : celle de la relation conjointe entre les deux adversaires.

Pour la seconde dimension, nous définirons plutôt un symptôme propre à l'agent : l'existence de ses émotions. Il est nécessaire de comprendre le rôle des émotions pour comprendre ce qu'exprime une action, qu'elle soit sportive ou artistique.

\subsection{Les actions sportives comme actions conjointes paradoxales}

La première dimension est l'expression d'une nécessité. Une relation entre deux êtres humains d'un certain type doit émerger nécessairement de l'action sportive. Comme le don ou la promesse, l'action sportive ne se résume 
pas à la motivation de l'agent, à ce qu'il fait physiquement pour agir, ou encore à ce qu'il ressent lorsqu'il agit. Car s'il en était ainsi, l'action sportive pourrait se concevoir comme celle d'un agent solitaire. Laction sportive n'existe que dans le cadre d'un engagement réciproque entre deux agents. L'action sportive est donc une "action conjointe » selon le paradigme énoncé par Sensevy (2011, p. 57). Cette expression désigne une certaine manière de coopérer. Il y a bien coopération selon les critères définis par Paternotte (2015, p. 199).

1. Il y a présence d'un but collectif: se confronter selon des règles établies.

2. L'intention collective est marquée par la stabilité des intentions individuelles : chacun s'engage à se confronter pendant une durée établie par les règles.

Cette manière d'agir ensemble présuppose la capacité d'agir intentionnellement, c'està-dire de savoir décrire ses propres actions de plusieurs façons. Pour agir intentionnellement, il est parfois nécessaire de savoir qu'en faisant X, je fais Y ou non Y. Nommons Y, le fait de se conduire en sportif. Je dois être capable de concevoir qu'en me dopant (en faisant X), simultanément je me retire de la catégorie des sportifs (je fais non Y), au minimum pour le laps de temps où le produit agira. C'est une conséquence logique de la relation qui existe entre deux sportifs. Cette évidence ne peut apparaître que si on sait ce qu'est réellement une action sportive. Et cela n'est pas toujours aisé. Car il est possible de se tromper. Ainsi, on peut croire que l'action sportive se résume à une action fondée sur la recherche d'une victoire sur un adversaire dans le cadre d'une pratique physique encadrée par des règles du jeu. C'est une description incomplète qui rate l'essentiel.

\subsection{L'aspect paradoxal de l'action sportive}

La coopération « n'exclut pas l'opposition, l'antagonisme " (Vernant, 1997, p. 160). Cela rend le concept en apparence paradoxal. Car la coopération, pour exister, nécessite "un bénéfice mutuel »(Paternotte, 2017, pp. 1-73). Or il s'avère que dans le cas du sport, la confrontation semble, de manière logique, produire simultanément un bénéfice pour l'un et une perte pour l'autre. Le paradoxe peut être résolu si on précise le caractère intentionnel de l'action sportive. L'action intentionnelle est caractérisée par «l'effet accordéon » décrit par Ascombe (2002, § 23-24). Cet effet est donné par la structure logique de l'action intentionnelle; en faisant quelque chose (lever le bras), je peux faire autre chose (donner un signal). Il en va ainsi du sens de l'action sportive : en voulant faire perdre mon adversaire, je tente de réaliser quelque chose qui nous fera gagner tous les deux.

Cette idée d'une action qui fait gagner deux sujets aux statuts complémentaires est le sens même de l'action conjointe sportive : elle n'existe que si la coopération entre les adversaires permet d'obtenir un gain pour tous et non pas pour le vainqueur seulement. La complémentarité des statuts des agents engagés dans l'action conjointe implique qu'il y ait un gagnant et un perdant. Il est même nécessaire qu'il y ait une véritable lutte pour qu'une action soit réellement sportive. Pour qu'existe une rencontre sportive, le perdant doit être réellement déçu.

Comment définir donc l'action sportive ? Selon le critère que nous venons de décrire, c'est une action conjointe éprouvée de manière paradoxale parce qu'elle est fondée à la fois sur la recherche d'une victoire dans le cadre d'une confrontation réglée éphémère $e t$ sur la nécessité de faire gagner aussi le perdant.

Les actions sportives sont caractérisées par des émotions appropriées. La déception du perdant (comme la joie du gagnant) doit faire place rapidement à une émotion d'une autre nature, celle d'avoir produit un lien entre deux êtres humains. Lien éphémère mais complexe, dans une lutte où plus rien ne compte hormis l'engagement pour la victoire, dans le respect de l'adversaire et des règles du jeu. C'est ce second temps du ressenti qui est le plus important et 
qui laisse au second plan le ressenti immédiat de la victoire ou de la défaite. Ce second temps qui résulte de l'engagement conjoint et symétrique dans le respect de l'autre est une condition nécessaire, ce n'est pas un bonus qui viendrait de temps à autre caractériser les actions sportives. La symétrie de l'engagement met hors sens et hors-jeu toute forme de tricherie.

L'engagement peut être total. C'est d'ailleurs ainsi que Queval (2004) définit le sportif, par une volonté de se dépasser. Les actions sportives de haut niveau semblent parfois " mimer» des actions de survie, mime dans lequel l'acteur n'est plus à distance de son jeu. Les recherches de la limite physique sont une des caractéristiques des actions du sportif. Voilà ce qui me motive, consciemment ou non, si je suis sportif : l'incertitude de l'engagement physique auquel va me contraindre mon adversaire pour que je réussisse à le vaincre. Et c'est ce qui distingue le sport d'un simple jeu : l'intensité de l'engagement physique. Que les sportifs soient poussés à atteindre leurs limites (sans les atteindre nécessairement) au moment de la compétition, c'est donc une condition logique de toute action sportive.

Mais de quelles limites s'agit-il ? Le sport est une expérience, non pas seulement de ce que nos corps peuvent faire, mais de tout ce que nous pouvons faire pour vaincre quelqu'un. Ce n'est pas une expérience qui résulte d'une fiction : celui qui en fait l'expérience ne ressent pas l'émotion de celui qui admire son champion en action, tranquillement assis sur son fauteuil.

\subsection{Les émotions dans l'activité sportive : une symptomatologie}

Tenter de comprendre ce qu'est une action sportive en faisant une analyse sociohistorique du sport, c'est négliger ce que Ruyer (1977, p. 289) appelle le montage psychologique, c'est-à-dire la "préparation mentale ou cérébrale qui enveloppe une action effective, la précède, et continue à la guider, à la contrôler, par facilitation, sélection, inhibition, en cours d'exécution ". On pourrait, à tort, croire pouvoir réduire les montages psychologiques aux influences sociales qui nous poussent à épouser une mode temporairement. Ruyer renverse l'idée selon laquelle la société influencerait en profondeur les montages psychologiques les plus représentatifs de l'espèce humaine. Au contraire, selon le philosophe (1977, p. 315), " les rapports sociaux sont à base d'appétit de reconnaissance par l'“Autre" [...]». Plus précisément, " la transaction sociale élémentaire, si elle est au-delà d'un simple passe-temps, et en deçà d'une intimité non manœuvrière, est un jeu, d'où chacun essaie de tirer autant d'avantages psychologiques que possible " (1977, p. 316). Cette idée inspirée des analyses transactionnelles de Berne (1966) met en lumière le moteur premier de toute interaction sociale : les motivations psychologiques. Mais l'originalité de Ruyer provient de sa capacité à relier les motivations psychologiques à des significations plus profondes, plus universelles. Sans aucun doute, dans la transaction sportive, il y a la volonté de dominer (l'autre et son propre corps) et d'être reconnu comme le vainqueur pour en tirer un bénéfice psychologique indéniable. Mais masquées derrière cette vitrine embellie par le culte de la domination, sorte de darwinisme social nécessaire au capitalisme, des ambitions bien plus profondes sont en action. Car, selon Ruyer, au-delà des jeux plus ou moins malhonnêtes liés à des transactions où on cache ses véritables intentions pour atteindre de petits bénéfices psychologiques, il existe des jeux où l'être humain se trouve " "en transaction" avec le principe de l'existence » (1977, p. 317). Comment découvrir de telles pratiques? "En considérant les attitudes qui sont le noyau des philosophies morales ou religieuses, comme des jeux avec l'univers ", écrit Ruyer (1977, p. 317). Sommes-nous si loin de la pratique sportive?

Le lien entre jeux des hommes et jeux de l'univers est essentiel car , «à l'arrière-plan des jeux avec les hommes, il y a le plus souvent une 
attitude envers l'univers qu'il y aurait avantage à dégager nettement, car consciente, elle assainirait l'attitude envers les hommes " (Ruyer, 1977, p. 318). Cette attitude, selon Koestler (2013, p. 193), est orientée par des émotions transcendantales qui ont toutes un dénominateur commun : " le sentiment de participation intégrante à une expérience qui dépasse les frontières du moi ». La tendance générale des individus est l'intégration à un groupe et de ce fait chaque individu renforce une composante sociale selon des modes plus ou moins pérennes. L'histoire en tant que discipline peut se saisir de cette évolution pour rendre compte des tendances sportives, la sociologie pour rendre compte de déterminismes de classes par exemple. Mais Koestler note que ce désir d'intégration peut fort bien ne pas être assouvi par l'appartenance à un groupe. La raison tient au fait que les motivations d'appartenance à un groupe sont souvent plus superficielles que celles qui tendent à faire éprouver à la personne le sentiment d'appartenir à quelque chose qui transcende les frontières du moi. L'homme est un être dont l'unité tient à sa capacité à participer à un ensemble qui le dépasse. Alors que « les émotions d'affirmation de soi répondent à l'activité corporelle» (Koestler, 2013, p. 193) et conduisent le sportif à une débauche d'énergie, « les émotions transcendantales sont essentiellement passives et cathartiques » (Koestler, 2013, p. 193). Or confiance en l'autre, en soi, empathie, participation, émerveillement, admiration, créativité sont liés à de telles émotions et se retrouvent aussi dans le sport. L'activité sportive est donc guidée par un montage mixte d'émotions, de sentiments qui ne dépendent pas du contexte. Voici ce qui rend nécessaire le symptôme émotionnel. Il y a quelque chose de décisif dans l'émotion de l'agent : elle rend compte de la présence d'une action juste. Ces états de conscience ne sont pas facultatifs. Quelqu'un qui donnerait l'impression d'accepter de s'engager sans manifester d'émotions ne ferait que simuler des actions sportives. Ceci réfute l'idée selon laquelle la finalité dans le sport se réduirait aux finalités sociales propres à une époque. Une finalité bien plus essentielle, bien plus primitive, incite le sportif à agir : " la finalité harmonie » (Louis, 2014, p. 148). En agissant, le sportif tente d'être en harmonie non seulement avec son corps, mais aussi avec l'adversaire qu'il tente de comprendre pour vaincre dans le respect des règles du jeu. Il entre aussi en harmonie avec des thèmes qui lui permettent de ressentir les propriétés esthétiques de ses actions. À propos des causes sociales, nous pouvons répéter ce que Ruyer (1952, p. 141) affirmait des causes physiques : "Des "causes", des occasions aussi insignifiantes que les divers agents de "détermination” biologique, mnémique ou inventive ne peuvent à elles seules rendre raison des immenses développements qu'elles amorcent. L'existence active, le développement des individus est une "succion" continue opérée par eux sur le monde trans-spatial, une "nutrition" au sens le plus général du mot. »

Récapitulons : agir sportivement revient à s'engager intensément physiquement, émotionnellement dans une action conjointe fondée à la fois sur la recherche d'une victoire dans le cadre d'une compétition éphémère et sur la nécessité de faire gagner aussi le perdant. Cette lutte constitue une œuvre réelle unique.

\section{QUELLE SORTE D'ÉUVRE LE SPORTIF ACCOMPLIT-IL ?}

Ce que le sportif accomplit est unique et non reproductible. L'œuvre du sportif s'inscrit dans l'ontologie événementielle de Davidson (1993, p. 284) : «Les événements sont des individus non répétables, datés, comme l'éruption particulière d'un volcan, la (première) naissance d'un individu [...]. » Ces événements sont des entités particulières, au même titre que les objets matériels. Certes, l'œuvre du sportif est plus proche de la performance, au sens où on l'entend en art contemporain, que de l'œuvre 
du peintre. Mais l'épreuve sportive est aussi un événement qui laisse des traces (le résultat) et en appelle d'autres (comme la revanche). Ces événements s'inscrivent le plus souvent dans un récit, celui du sportif, celui d'un sport ou celui d'une nation. Pourquoi l'œuvre du sportif est-elle non reproductible alors qu'une même œuvre musicale peut être reproduite ? Cela tient essentiellement au fait que la compétition est un moment de partage qui a un rapport étroit avec notre manière de ressentir l'intensité et la fugacité de la vie. Certes, ce sentiment peut exister également lorsqu'on se laisse prendre par une œuvre de fiction. Mais ce que le sport crée de toutes pièces, c'est la réalité d'une fin imminente. Cette mort symbolique est parfois accompagnée d'une telle souffrance physique lors de l'effort que l'imminence de la mort ne semble pas seulement symbolique.

Ensuite, l'action sportive exprime aussi ce qu'est chacun des adversaires : elle l'exprime comme une signature produite non par leur main mais par leur corps tout entier et elle exprime également comme une co-signature, irréductible à un simple couple de deux signatures, ce que sont les deux adversaires ensemble, dans le cadre d'un jeu dont les règles sont bien connues.

Enfin, l'événement, aussi recherché soit-il, aussi spectaculaire puisse-t-il être, est un aboutissement. Il est le reflet de l'éducation que le sportif a reçue pour se comporter lors de l'événement. L'événement sportif est alors une sorte de célébration qui ne peut être réellement comprise que lorsqu'on cerne les vertus que le sportif doit avoir pour accomplir son œuvre et qu'on saisit la complémentarité des sportifs en compétition.

\section{De la nÉCEssité éthique de soutenir UNE DÉFINITION LOGIQUE ET NON SOCIOLOGIQUE DE L'ACTIVITÉ SPORTIVE : RELATIVISME ET OBJECTIVITÉ DES APPROCHES}

L'approche socio-historique aboutit au relativisme. Pourquoi ? Selon Pouivet (1999, p. 64), il existe deux sortes de mentalisme. Le plus connu est le mentalisme internaliste, conception qui donne au sujet toute l'autorité pour dire quel est le sens de ses paroles ou de ses pensées. Ainsi, nulle objectivité n'est possible puisque le sujet, en son for intérieur, forge le sens de ses pensées et de ses paroles. Et le sens des paroles n'est défini que relativement au sujet. Mais il y a un autre mentalisme, externaliste pourrait-on dire, c'est le mentalisme sociologique. Comme l'écrit Pouivet (1999, p. 64), le mentalisme sociologique est cette conception qui « dit parfois que pour savoir ce que sont certains objets, comme les œuvres d'art ou certaines conceptions religieuses ou morales, on doit connaitre la mentalité d'un groupe, d'une époque, d'une nation, etc. [...] ». Or nos actions sont intelligibles dès que nous comprenons les raisons qui les motivent. Et comme pour les comportements artistiques, les conduites sportives ne peuvent être comprises, en tant que telles, que si nous admettons qu'au-delà des simples intentions de boire, gagner de l'argent, communiquer, jouer, il existe des intentions sportives. Ceci signifie que ce qui organise rationnellement l'action est alors spécifiquement sportif et non réductible à d'autres intentions qui seraient les avatars de nos conduites sociales. Ces actions sportives peuvent être alors décrites de manière non relative. Est sportif, non pas celui qui se conduit bien au regard des normes sociales en vigueur (être un modèle pour le capitalisme qui se nourrit de compétition) mais celui qui se conduit de manière vertueuse au regard de l'intention de produire une œuvre sportive. Si le sportif qui se comporte exceptionnellement n'est au final que le bon exemple relativement à une norme 
somme toute contingente et discutable, alors le sportif ne fait preuve que de compétences, comme n'importe quel autre animal particulièrement adapté à son environnement. $\mathrm{Si}$ au contraire, comme nous allons le défendre, l'action sportive existe quand le pratiquant fait preuve de vertus alors l'éthique de la pratique résulte de la définition du sport et elle n'est pas relative aux mœurs de l'époque.

Deux aspects de notre étude paraissent cependant menacer l'objectivité de notre définition : la référence à l'intention de produire une œuvre et la référence au ressenti nécessaire d'un engagement complet. Or, en sciences humaines, la crainte est grande de devoir admettre que la subjectivité est le moteur de l'agentivité. Car le projet de rendre compte objectivement des phénomènes sociaux est difficilement compatible avec l'idée que les actions des agents sont caractérisées par un aspect irrémédiablement propre à l'agent.

Faire appel aux intentions pour décrire l'activité humaine passe parfois pour un manque d'objectivité. L'intentionnalité est prise parfois comme un retour à la subjectivité. Ce serait le cas si nous définissions l'intention dans le cadre d'un mentalisme qui confond intention et intériorité, faisant de l'intention une sorte d'action interne et privée au sujet. Ainsi, nous attribuerions la propriété d'être sportive à une action dans le cas où le pratiquant serait dans un état mental particulier. La propriété serait alors relative à un état interne du sportif. Ce n'est pas le cas dans cette étude. «L'intention est ce qu'on présuppose pour rendre raison de certains comportements " (Pouivet, 1999, p. 64). L'intentionnalité qu'on attribue à des agents, c'est « une structure noétique, c'est-àdire un ensemble de croyances et de relations logiques et épistémiques que ces croyances entretiennent "(Pouivet, 1999, p. 65). Ainsi, on peut attribuer à un agent une intention de se comporter en sportif au vu d'un ensemble de croyances, actions, réactions, émotions du pratiquant.
Nous avons soutenu que l'agent, par son engagement dont lui seul mesure l'intensité véritable, sait s'il se comporte comme un sportif. Mais ce n'est là qu'un symptôme qui doit être pris en compte comme lorsque le médecin nous demande sur une échelle de 1 à 10 le degré de souffrance qui nous est infligé par la maladie. Nous avons soutenu également que le sportif devait ressentir des émotions appropriées : celui qui se conduit en montrant seulement à son adversaire vaincu qu'il tient là tout ce qu'il espérait, à savoir vaincre, montre de manière objective qu'il n'est pas encore sur la voie du sport. La joie de gagner est d'autant plus grande que l'adversité a été importante. La joie de gagner ne doit donc pas effacer la joie d'avoir gagné avec un adversaire qui a montré des qualités sans lesquelles le sport et la victoire n'existeraient pas. Le relativiste tenterait sans doute de mettre en doute l'objectivité de notre description en insistant sur le fait que (dans notre description) l'action sportive doit produire un effet (émotionnel et physique) sur le pratiquant qui juge alors lui-même de la qualité de son action. Il est certain qu'un effet doit être produit sur les deux adversaires. Mais l'aspect relatif n'est ici qu'apparent car seuls les deux adversaires sont concernés par l'action. En tant que spectateur, je peux juger de la qualité de l'œuvre produite mais je peux me tromper sur la réalité de l'engagement et sur la sincérité des adversaires. Néanmoins, la possibilité de se tromper n'implique pas la relativité des propriétés qui sont l'objet du jugement.

\section{RÉALITÉ DE L'đEUVRE DU SPORTIF COMME PRODUIT DE L'EXERCICE DE VERTUS HUMAINES}

Selon Pouivet (2006, p. 86), « les vertus sont des dispositions qui sous certaines conditions font réagir quelque chose conformément à sa nature ». L'art et l'humain, dit R. Pouivet (2017, p. 17), « sont ainsi des réalités métaphysiquement liées, dans leur existence même, et selon leurs natures propres. D'un côté, la nature 
humaine trouve sa perfection dans la production artefactuelle et artistique. Et de l'autre, la perfection de l'art se trouve dans des biens proprement humains qui sont ses finalités. » $\mathrm{La}$ défense du réalisme est ici la même que pour celle de l'esthétique. Elle aboutit à une éthique des vertus. Le bien proprement humain qui est caractéristique du sport, c'est la coopération paradoxale. Pour que ce type de coopération existe, des vertus telles que la prudence, l'honnêteté, le courage, la persévérance, la justice, etc., sont nécessaires. Ces vertus se développent alors par la sollicitation arétique produite par les œuvres des sportifs et les propriétés de leurs actions.

Or, que ce soit dans le cadre de l'esthétique ou du sport, attribuer des propriétés " (à des œuvres d'art ou à autre chose) suppose une classe de "percepteurs" appropriés, des personnes qui possèdent elles-mêmes des qualités grâce auxquelles ces propriétés sont perceptibles » (Pouivet, 2006, p. 139). Il est nécessaire que certaines personnes aient les bonnes réactions en appréhendant la réalité sous la bonne description pour qu'existent ces propriétés sportives. La description des propriétés sportives dit comment est le monde pour ceux qui ont des réactions appropriées. Les émotions du sportif (et du spectateur), lorsqu'elles sont appropriées, témoignent de l'existence de propriétés sportives des actions.

Ce qui est donc premier dans l'ordre d'un principe éducatif, c'est l'éducation au sport et non pas l'éducation par le sport. Être bon dans le domaine artistique ou dans le domaine du sport, c'est faire preuve d'une forme d'humanité qui s'apprend. Cette forme d'humanité n'a pas toujours existé. En ce sens, comme «il n'y a pas d'esthétique sans métaphysique " (Pouivet, 2006, p. 232), il ne saurait y avoir de discours sur le sport sans métaphysique. Les vertus sportives ont un aspect métaphysique : les émotions, en tant qu'aspects de nos vertus expriment notre nature, nous permettent de connaître notre monde et de reconnaître la vraie nature de ceux qui agissent d'une certaine manière, celle qui fait de nous des sportifs.

\section{CONCLUSION}

En faisant du sport un marqueur de notre société, l'approche socio-historique élimine toute possibilité de concevoir le sport comme une activité de production d'œuvres qui, par nature, permettraient de développer certaines vertus : confiance en soi, confiance dans les autres, émotions esthétiques, goût de la persévérance. Mais l'approche élimine également la possibilité de comprendre que les propriétés de l'œuvre sont bien réelles. Ces propriétés n'existent que parce que certaines personnes éduquées sont en mesure de comprendre ce qu'est pratiquer un sport. En faisant du pratiquant un simple relais de croissance des valeurs en vogue dans la société, l'approche socio-historique se coupe de la possibilité de comprendre ce qui fait exister les œuvres sportives : les vertus et non seulement les compétences des pratiquants.

Le sportif est un homme qui s'engage : cet engagement consiste à rechercher la voie d'un accomplissement personnel qu'il ne peut réaliser seul. Les actions sportives sont symptomatiques d'êtres humains qui cherchent à se réaliser ensemble en tant qu'être humain. Et cette réalisation proprement humaine ne peut aboutir sans une éducation précise, utile non seulement pour que le sportif maitrise son corps et ressente des émotions appropriées, mais aussi pour développer les vertus nécessaires à la compréhension et à l'accomplissement d'une nouvelle relation qui vient enrichir l'humanité.

\section{BibLIOGRAPHIE}

Anscombe, G.E.M. (2002). Lintention. Traduit de l'anglais par M. Maurice \& C. Michon. Paris : Gallimard Berne, É. (1966). Des jeux et des hommes. Paris : Stock Brohm, J.-M. (1976). Sociologie politique du sport, Paris : Jean-Pierre Delarge. 
Chauvier, S. (2007). Qu'est-ce qu'un jeu ? Paris : Vrin.

Davidson, D. (1993). Actions et événements. Paris : PUF.

Foucault, M. (1969). L'archéologie du savoir. Paris : Gallimard.

Gillet, B. (1949). Histoire du sport. Paris : PUF.

Gleyse, J. (2015). Archéologie. In B. Andrieu (dir.), Vocabulaire international du sport, tome 1. Paris : L'Harmattan.

Guay, D. (1993). La culture sportive. Paris : PUF.

Haumesser, M. (2008). De quelle matière le sport est-il fait? In D. Moreau \& P. Taranto (dir.). Activités physiques et exercices spirituels. Paris : Vrin.

Ingold, T. (2013). Marcher avec les dragons. Traduit de l'anglais par P. Madelin. Bruxelles : Zones sensibles.

Ingold, T. \& Descola, P. (2014). Etre au monde, quelle expérience commune? (avec Philippe Descola). Traductions de l'anglais par B. Fau. Lyon : Presses universitaires de Lyon, coll. « Grands débats : mode d'emploi ".

Jeu, B. (1993). Le sportif, le philosophe, le dirigeant. Lille : Presses universitaires de Lille.

Koestler, A. (2013). Le cheval dans la locomotive : le paradoxe humain. Traduit de l'anglais par G. Fradier. Paris : Les Belles Lettres.

Lesage, T. (2015). L'analyse des filiations sportives à la confluence des sciences sociales et des sciences de l'évolution : un nouveau champ d'investigation. In A. Tatu-Colasseau, Sociologie(s) du sport : analyses francophones et circulation des savoirs (Emplacements du Kindle 3455-3458). Paris : L'Harmattan.

Louis, F. \& Louis J.-P. (2014). La philosophie de Ruyer. Paris : Vrin.

Moreau, D. \&t Taranto, P. (2008). Activités physiques et exercices spirituels. Essais de philosophie du sport. Paris : Vrin.

Ollier, F. (2010). Quel sport?, n 12-13.
Parlebas, P. (1985). La dissipation sportive., Culture technique, $\mathrm{n}^{\circ} 13$.

Parlebas, P. (2015). Le sport et l'action motrice sous le regard des sciences sociales. In A. Tatu-Colasseau, Sociologie(s) du sport : analyses francophones et circulation des savoirs, (Emplacements du Kindle 21572158). Paris : L'Harmattan.

Partenotte, C. (2017). Agir ensemble : fondements de la coopération. Paris : Vrin.

Pouivet, R. (1999). L'ontologie de l'œuvre d'art. Nîmes : Jacqueline Chambon.

Pouivet, R. (2006). Le réalisme esthétique. Paris : PUF.

Pouivet, R. (2017). L'art et le désir de Dieu. Rennes, PUR.

Rauch, A. (1970). Revue Les Cahiers scientifiques de l'éducation physique, mars.

Ruyer, R. (1952). Néo-finalisme. Paris : PUF;

Ruyer, R. (1977). La gnose de Princeton. Paris : Fayard.

Sensevy, G. (2011). Le sens du savoir. Éléments pour une théorie de l'action conjointe en didactique. Bruxelles : De Boeck.

Terret, T. (2010). Histoire du sport. Paris : PUF.

Queval, I. (2004). S'accomplir ou se dépasser. Paris : Gallimard.

Vernant, D. (1997). Du discours à l'action. Paris : PUF.

Vieille-Marchiset, G. \& Tatu-Colasseau, A (2015). Genèse et circulation des savoirs en sociologie du sport de langue française. In A. Tatu-Colasseau, Sociologie(s) du sport : analyses francophones et circulation des savoirs (Emplacements du Kindle 40-41). Paris : L'Harmattan.

Vigarello, G. (2003). Systèmes de sports, systèmes concurrents. In Travailler avec Bourdieu. Paris : Flammarion.

Wittgenstein, L. (2004). Recherches philosophiques. Paris : Gallimard.

\section{Resumen: La esencia del deporte. Por un enfoque antropológico y metafísico}

Se trata de dar un análisis conceptual y una definición esencialista de la acción deportiva. Por otra parte sostenemos que las propiedades que se les atribuyen a las acciones deportivas son reales. Nos oponemos a estas dos concepciones:

CSH: concepción sociohistórica. En tal concepción, el deporte se reconoce como el conjunto de prácticas físicas emergentes, en evolución, que desaparecen, que encuentran una función y un significado en un contexto social específico.

CRE: concepción realista y esencialista del deporte. En esta perspectiva, practicar deporte consiste en participar físicamente, emocionalmente, intensamente, en el contexto de una competencia que resulta de una acción conjunta paradójica y produce un trabajo único.

El deporte refleja un rasgo de la naturaleza humana, la capacidad de cooperar de una nueva manera a la forma en que hemos aprendido a cooperar en el desarrollo de actos de nuevos lenguajes 
y específicamente humanos. Debemos aprender, no en qué deporte puede ser útil para, o cual es el valor, sino simplemente en qué es el deporte.

Palabras clave: deporte, realismo, esencialismo, filosofía, acción conjunta

\section{Zusammenfassung: Das Wesen des Sports. Für einen anthropologischen und metaphysischen Ansatz}

Ziel ist es, eine konzeptionelle Analyse und eine essentialistische Definition von sportlichem Handeln zu liefern. Andererseits werden wir argumentieren, dass die dem Sport zugeschriebenen Eigenschaften real sind. Wir stellen diese beiden Vorstellungen einander gegenüber:

CSH: Sozio-historische Konzeption. In einer solchen Konzeption wird Sport als die Gesamtheit der anerkannten körperlichen Praktiken verstanden, die entstehen, sich entwickeln, verschwinden und eine Funktion und Bedeutung in einem präzisen sozialen Kontext finden.

CRE: realistische und essentialistische Auffassung von Sport. Aus dieser Perspektive besteht die Ausübung des Sports darin, sich körperlich, emotional und intensiv an einem Wettkampf zu beteiligen, der aus einer paradoxen gemeinsamen Aktion resultiert und ein einzigartiges Produkt hervorbringt. Der Sport spiegelt ein Merkmal der menschlichen Natur wider, nämlich die Fähigkeit zur Zusammenarbeit auf eine neue Art und Weise, ebenso wie wir gelernt haben, bei der Entwicklung neuer und insbesondere menschlicher Sprechakte zusammenzuarbeiten. Wir müssen lernen, nicht wodurch der Sport für uns nützlich sein kann oder wodurch er einen Wert hat, sondern einfach was Sport ist.

Schlagwörter: Sport, Realismus, Essentialismus, Philosophie, gemeinsame Aktion

\section{Riassunto: L'essenza dello sport. Per un approccio antropologico e metafisico}

Si tratta di fare un'analisi concettuale e una definizione essenzialista dell'azione sportiva. D'altra parte, noi sosteniamo che le proprietà che si attribuiscono a delle azioni sportive sono ben reali. Noi proponiamo queste due concezioni:

CSS: concezione socio-storica. In una tale concezione, lo sport è concepito come l'insieme delle pratiche fisiche riconosciute come, nascenti, evolventi, scomparenti, trovanti una funzione e un senso con un contesto sociale preciso.

CRE: concezione realista ed essenzialista dello sport. In questa prospettiva, praticare dello sport consiste nell'impegnarsi fisicamente, emozionalmente, in maniera intensa, nel quadro di una competizione che risulta da un'azione congiunta paradossale e che produce un'opera unica.

Lo sport riflette un tratto della natura umana, quello di una capacità di cooperare in una maniera nuova, con la maniera con cui abbiamo imparato a cooperare per elaborare degli atti di linguaggio nuovi e specificamente umani. Bisogna imparare, non in cosa lo sport può essere utile, oppure in cosa ha di valore, ma semplicemente ciò che è lo sport.

Parole chiave: azione congiunta, essenzialismo, filosofia, realtà, sport 
